\title{
Left-Right Determination: Involvement of Molecular Motor KIF3, Cilia, and Nodal Flow
}

\author{
Nobutaka Hirokawa, Yosuke Tanaka, and Yasushi Okada \\ Department of Cell Biology and Anatomy, University of Tokyo, Graduate School of Medicine, \\ 7-3-1 Hongo, Tokyo, 113-0033 Japan \\ Correspondence: hirokawa@m.u-tokyo.ac.jp
}

\begin{abstract}
Mammalian left-right determination is a good example for how multiple cell biological processes coordinate in the formation of a basic body plan. The leftward movement of fluid at the ventral node, called nodal flow, is the central process in symmetry breaking on the left-right axis. Nodal flow is autonomously generated by the rotation of posteriorly tilted cilia that are built by transport via KIF3 motor on cells of the ventral node. How nodal flow is interpreted to create left-right asymmetry has been a matter of debate. Recent evidence suggests that the leftward movement of sheathed lipidic particles, called nodal vesicular parcels (NVPs), may result in the activation of the noncanonical hedgehog signaling pathway, an asymmetric elevation in intracellular $\mathrm{Ca}^{2+}$ and changes in gene expression.
\end{abstract}

\begin{abstract}
lthough the human body is apparently Abilaterally symmetrical on the surface, the visceral organs are arranged asymmetrically in a stereotyped manner. The heart, spleen, and pancreas reside on the left side of the body, whereas the gall bladder and most of the liver are on the right side (Fig. 1A). Because the human body is formed from a spherically symmetrical egg (oocyte), symmetry breakdown is one of the fundamental processes of development.

In many lower vertebrates and invertebrates, eggs are asymmetrical even before fertilization (as in Drosophila [Gilbert 2003]). In some organisms, such as fish and frog, the dorsoventral (DV) and anteroposterior (AP) axes are determined at fertilization by the distribution of the yolk and the entry position of the
\end{abstract}

sperm (Gilbert 2003). In human, mouse, and other mammals, the embryo is initially cylindrically symmetrical when it implants itself into the wall of the uterus. The DV axis is first to be specified as the proximal-distal axis from the implantation site. Subsequently, the AP axis is arbitrarily determined in the plane perpendicular to the DV axis (Alarcon and Marikawa 2003; Beddington and Robertson 1999). In either case, $L / R$ is thus the last axis to be determined, and needs to be consistent with the preceding DV and AP axes. Because the chirality of the body is predetermined by chiral molecules, such as amino acids and nucleic acids, the laterality or orientation of the $\mathrm{L} / \mathrm{R}$ axis is established theoretically or potentially once the AP and DV axes are determined. The problem here is how this

Editors: Bruce Bowerman and Rong Li

Additional Perspectives on Symmetry Breaking in Biology available at www.cshperspectives.org

Copyright (C) 2009 Cold Spring Harbor Laboratory Press; all rights reserved; doi: 10.1101/cshperspect.a000802

Cite this article as Cold Spring Harb Perspect Biol 2009;1:a000802 

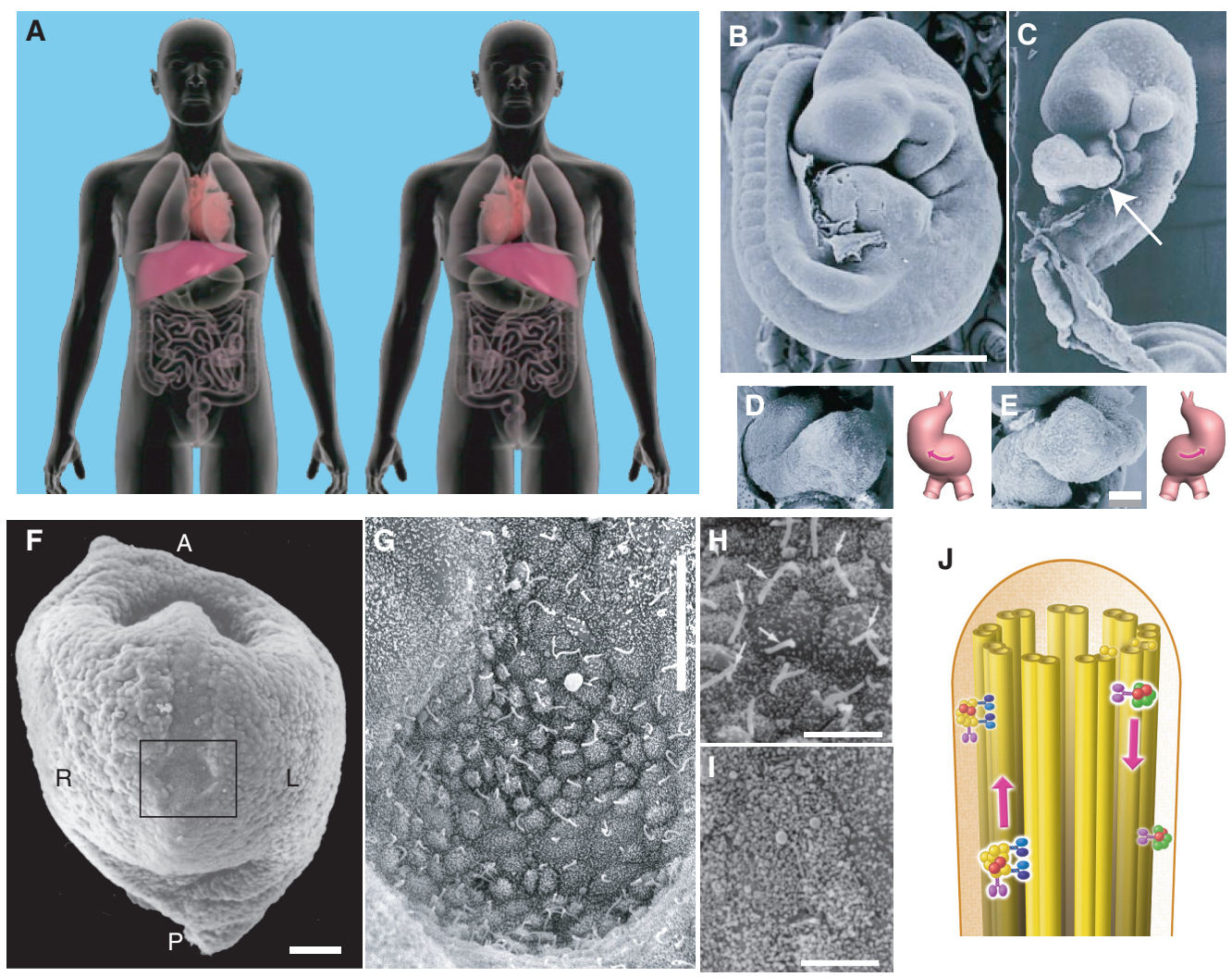

Figure 1. (A) Left-right asymmetric arrangements of internal organs in the human body. (Left) Normal arrangement (situs solitus). Most humans $(>99 \%)$ have the heart on the left side and the liver on the right side. (Right) Mirrored arrangement (situs inversus). Half of patients with Kartagener's syndrome have this arrangement, whereas the remaining patients are normal. Therefore, the left-right bilateral symmetry is randomly broken in this disease. $(B-E)$ Scanning electron micrographs of wild-type $(B, D)$ and $K_{i f 3} b^{-/-}$ $(C, E)$ mouse embryos. $(B, C)$ Full-length images. Wild-type embryos at this stage have already turned with a right-sided tail $(B)$, whereas $K i f 3 b^{-/-}$embryos remain unturned $(C)$. In panel $C$, the dilated pericardial sac has been removed, and the heart loop is inverted (arrow). $(D, E)$ Higher-magnification images and schematic representations of the heart loops showing a normal loop in the wild-type embryo $(D)$ and an inverted loop in the mutant embryo $(E)$. $(F-I)$ Scanning electron micrographs of a mouse node. $(F)$ Low-magnification view of a mouse embryo at 7.5 days postcoitum. Reichert's membrane is removed, and the embryo is observed from the ventral side. The node is indicated by a black rectangle. The orientation is indicated in the panel as anterior (A), posterior $(\mathrm{P})$, left $(\mathrm{L})$, and right $(\mathrm{R})$. Scale bar $=100 \mu \mathrm{m}$. $(G)$ Higher-magnification view of the mouse node. The orientation is the same as in panel $A$. Scale bar $=20 \mu \mathrm{m}$. $(H)$ Higher-magnification view of the nodal cilia (arrows) and nodal pit cells. Scale bar $=5 \mu \mathrm{m}$. $(I)$ Nodal pit cells of Kif3 $3 b^{-/-}$embryos. Nodal cilia are absent in these genetically manipulated embryos. $(J)$ Intraflagellar transport. Protein components in the cilia and flagella are transported by KIF3A/B complexes (light and dark blue) along the doublet microtubules of the axoneme. (Panels $A$ and $J$ were reproduced with permission from JT Biohistory Research Hall/TokyoCinema. B-I were modified from Nonaka et al. 1998, Okada et al. 2005, and Hirokawa et al. 2006, with permission.) 
potentially established laterality is materialized through developmental events. This mechanism is still totally unknown for the invertebrates. However, recent studies of mouse embryo clarified the $\mathrm{L} / \mathrm{R}$ determination mechanism in mammalian embryos (Hirokawa et al. 2006).

Until recently, little was known about the mechanism for breaking L/R symmetry. The initial clue to tackling this question was a human genetic disease called Kartagener's syndrome. Approximately half of these patients have their organs in the reversed orientation (situs inversus). Thus, the $\mathrm{L} / \mathrm{R}$ determination is randomized in this syndrome. Patients with Kartagener's syndrome also suffer from sinusitis and bronchiectasis (Kartagener 1933). A number of male patients with Kartagener's syndrome are sterile. Affected individuals have immotile sperm and defective cilia in their airway (Afzelius 1976). Airway cilia and sperm from these patients have abnormal ultrastructures. Specifically, the axonemes, the interior supramolecular complexes that produce the movement of cilia and flagella, lack dynein arms, the molecular motors required for cilia and flagella motility (Afzelius 1976). However, the relationships between these phenotypes and the causes of situs inversus remained unknown for more than 20 years.

Approximately 10 years ago, molecular biological studies identified several genes that are asymmetrically expressed in the $\mathrm{L} / \mathrm{R}$ orientation before $\mathrm{L} / \mathrm{R}$ asymmetric morphogenesis of the embryo. Morphological L/R asymmetry first becomes apparent with the orientation of the heart-tube loop (Fig. 1B,D) (Kaufman 1992), but initial L/R asymmetric gene expression precedes the morphological changes. In early embryos (7.5 days for mice) at the stage of somatogenesis, genes such as Lefty-2 (Ebaf), Nodal, and Pitx2 are expressed in the left lateral plate mesoderm, a structure located on the side of the embryos (Capdevila et al. 2000; Hamada 2002; Harvey 1998; Levin 2005; Yost 1999). However, the upstream phenomena that cause asymmetrical expression of these genes remain enigmatic.

Many studies have suggested that the so-called node, a concave triangular region transiently formed during gastrulation at the ventral midline surface of early embryos, is important for $\mathrm{L} / \mathrm{R}$ determination (Harvey 1998). When viewed from above the ventral side, the node of mouse embryos appears as a roughly triangular depression with the apex pointed toward the anterior (Fig. 1F,G), and it is $50-100 \mu \mathrm{m}$ in width and $10-20 \mu \mathrm{m}$ in depth. This nodal pit is covered by Reichert's membrane, and the cavity is filled with extraembryonic fluid. The ventral embryonic surface of the nodal pit consists of an epithelial sheet of a few hundred monociliated cells (nodal pit cells).

Nodal pit cells have one or sometimes two cilia that appear as rodlike protrusions approximately $5 \mu \mathrm{m}$ in length and $0.3 \mu \mathrm{m}$ in diameter (Fig. 1G,H). Because Kartagener's syndrome suggests a potential link between cilia motility and $\mathrm{L} / \mathrm{R}$ determination, the cilia in the node have been postulated to be motile and responsible for $\mathrm{L} / \mathrm{R}$ determination.

However, the ultrastructure of these nodal cilia is similar to that of immotile primary cilia. In motile cilia and flagella, nine pairs of doublet microtubules are arranged longitudinally along the axes (Fig. 2A) (Satir and Christensen 2007). Adjacent pairs of doublet microtubules are connected by dynein arms, which generate the motility of cilia and flagella. In addition, there are two microtubules in the center of cilia and flagella, referred to as the central pair, which define the direction of the beating plane. This essential central pair of microtubules is missing in primary cilia. Similar to other immotile primary cilia, the monocilia of nodal pit cells lack the central pair of microtubules and thus have a $9+0$ microtubule arrangement. Therefore, based on their ultrastructure and initial video-microscopic observations, nodal monocilia were originally considered immotile (Bellomo et al. 1996).

Through studies of the flow of materials within cells, we serendipitously found that nodal cilia are actually motile and vigorously rotating. This rotation generates the leftward flow of extraembryonic fluid in the nodal pit. The directionality of this flow, termed nodal flow, determines laterality. Thus, quite 
N. Hirokawa, Y. Tanaka, and Y. Okada

A
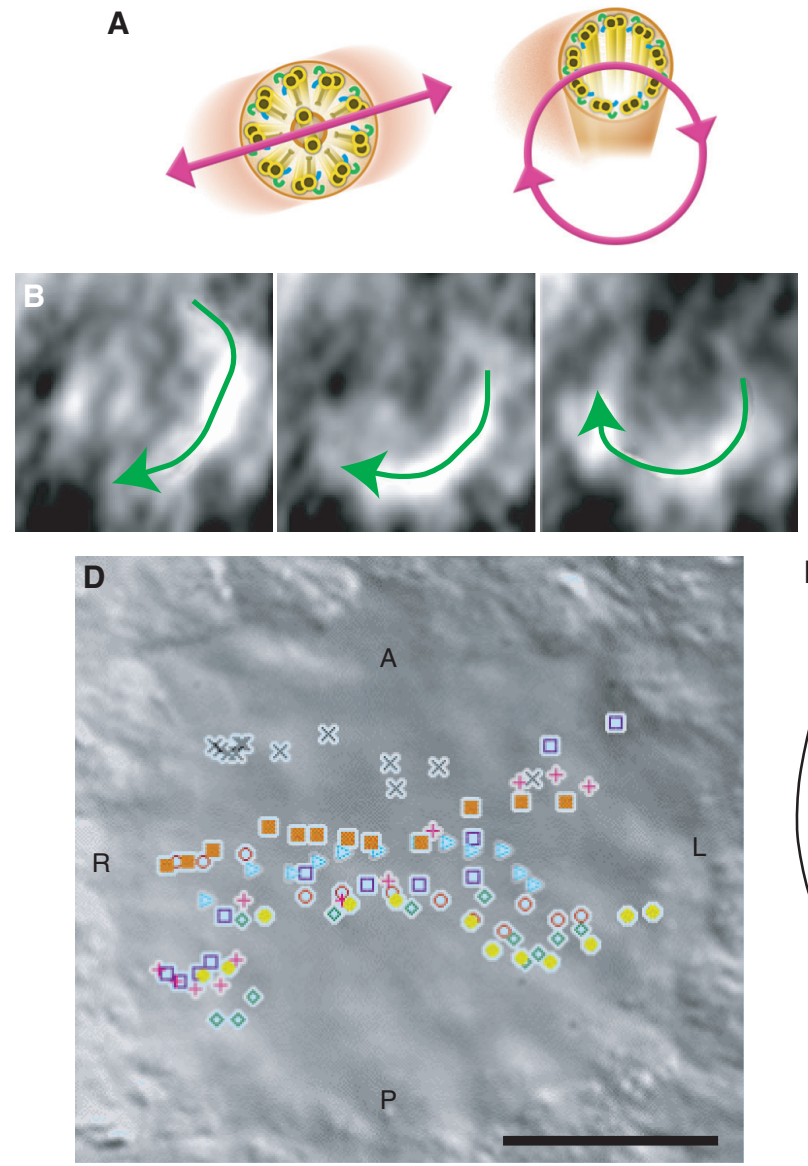

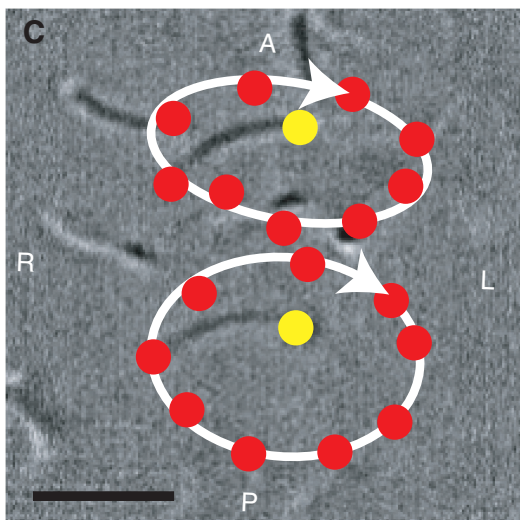

E

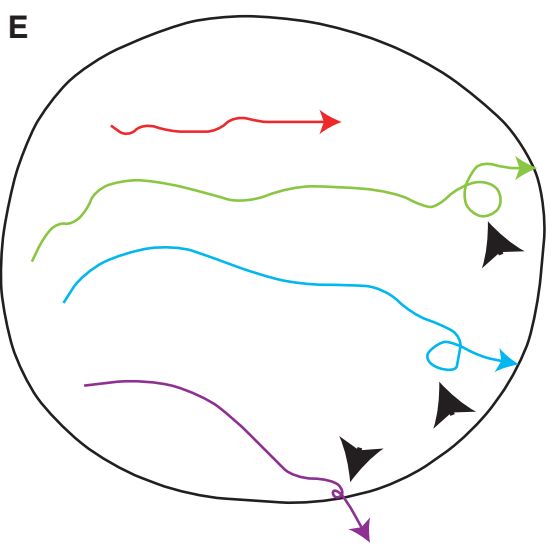

Figure 2. (A) Ultrastructures of normal cilia and primary cilia. (Left) Normal cilia and flagella have nine pairs of doublet microtubules (yellow) and two central microtubules (yellow). Adjacent doublet microtubules are connected with dynein motors (blue and green). The orientation of the central pair of microtubules is considered to determine the beating plane (purple). (Right) The central pair of microtubules is missing in immotile primary cilia and nodal cilia. In nodal cilia, the dynein motors remain in a chiral arrangement and produce a rotation-like movement (purple). $(B-E)$ Rotation of nodal cilia and leftward nodal flow. The images are views from the ventral side. The orientation is indicated in the panels as anterior (A), posterior $(\mathrm{P})$, left (L), and right (R). (B) Trajectory of a fluorescent bead attached to the tip of a nodal cilium (see online Movies 1 and 2 at http://beta.cshperspectives.cshlp.org). Three consecutive video frames with 33-ms exposures at 16-ms intervals (interlaced scan) are shown. The moving bead produces arc-shaped images (traced by green arrows). The beads rotate clockwise when viewed above the node. $(C)$ Trajectories of the tips of nodal cilia traced from a high-speed video sequence (500 frames/s) (see online Movie 3 at http:// beta.cshperspectives.cshlp.org). The red circles show the positions of the ends of the cilia at 10-ms time intervals, and the yellow circles show the positions of the roots of the cilia. The white ellipses show the trajectories of the tips. Scale bar $=5 \mu \mathrm{m}$. (D) The positions of beads that entered the node from the right edge traced for 4 seconds at 0.33 -second intervals. Different symbols indicate different beads. Most beads go straight to the left edge of the node. Scale bar $=20 \mu \mathrm{m}$. (E) The trajectories of four beads selected to illustrate the streamline of the nodal flow. The flow is mostly laminar and straight in the middle of the node, but often makes small vortices near the left edge (arrowheads). (Panel A was reproduced with permission from JT Biohistory Research Hall/TokyoCinema. B-E were modified from Okada et al. [1999, 2005] with permission.) 
unexpectedly, a physical process, fluid flow, was identified as the initial L/R symmetry-breaking event. In this review, we first summarize the discovery of nodal flow and then discuss how this leftward linear flow is generated in a fluid dynamic manner by the rotational movement of cilia. We further discuss the mechanisms by which $\mathrm{L} / \mathrm{R}$ asymmetry is determined by this nodal flow.

\section{THE DISCOVERY OF NODAL FLOW}

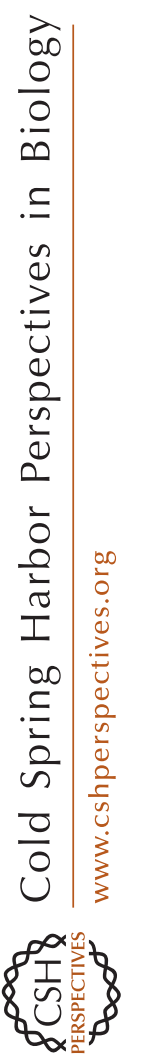

The discovery of nodal flow generated by the rotation of cilia, which plays a fundamental role in L/R asymmetry, was based on studies of molecular motors. Cells transport and sort various proteins and lipids after synthesis to their correct destinations at appropriate velocities. This is true for all cell types, including both nonpolarized cells (e.g., fibroblasts) and polarized cells (e.g., neurons and epithelial cells) (Hirokawa 1998; Hirokawa and Noda 2008; Hirokawa and Takemura 2005). Neurons, the units of brain wiring, are highly polarized cells comprising dendrites, a cell body, and a long axon (sometimes longer than $1 \mathrm{~m}$ ) extending along the direction of impulse propagation (see Tahirovic and Bradke 2009). Most proteins required in the axon and synaptic terminals need to be transported down the axon after their synthesis in the cell body, and this intracellular transport is therefore fundamental for neuronal morphogenesis, functions, and survival. Because similar mechanisms are observed in all other cells, neurons serve as a good model system for studying the general mechanisms of organelle transport.

Microtubules, comprising tubelike structures with a diameter of $25 \mathrm{~nm}$, serve as the rails for transport over long distances and are arranged regularly in many kinds of cells. In nerve axons, the microtubules are arranged longitudinally with their plus ends (fast-growing ends) pointing distally, whereas in epithelial cells, the microtubules are organized longitudinally with their plus ends pointing toward the basolateral surface. In nonpolarized cells, such as fibroblasts, microtubules radiate from a microtubule-organizing center located close to the nucleus with their plus ends pointing toward the cell periphery. Kinesin superfamily proteins (KIFs) and cytoplasmic dynein are the motor proteins for the transport along microtubules (Aizawa et al. 1992; Hirokawa 1998; Hirokawa and Takemura 2005). There are $45 \mathrm{KIF}$ genes identified in mammalians such as human and mouse (Miki et al. 2001; Miki et al. 2005), and distinct kinds of KIFs transport various cargos, such as synaptic vesicle precursors (KIF1A/KIF1B $\beta$ ); mitochondria (KIF1B $\alpha /$ KIF5s); NMDA-type (KIF17) and AMPA-type (KIF5s) glutamate receptors, and $\mathrm{N}$-cadherin/beta-catenin-containing vesicles (KIF3) (Hirokawa and Noda 2008; Hirokawa and Takemura 2005).

Among the KIFs, the KIF3 motor comprises a heterodimer of the motor proteins KIF3A and KIF3B and an associated protein, KAP3, which binds to the tail ends of KIF3A and KIF3B. To analyze the function of KIF3 in vivo, our group and others independently generated Kif $3 a$ and Kif $3 b$ knockout mice. These mutant mice show embryonic lethality. Studies on Kif $3 a$ and Kif $3 b$ knockout mouse embryos revealed that approximately $50 \%$ of KIF3Adeficient and KIF3B-deficient mice show reversed heart loops, whereas the rest are normal (Fig. 1C,E). To determine the positions of KIF3A and KIF3B in the signaling cascade for $\mathrm{L} / \mathrm{R}$ determination, we examined the expression of Lefty-2, one of the earliest left-defining genes. Although Lefty-2 is expressed exclusively on the left side of wild-type embryos, its expression is either mostly bilateral or absent in KIF3A-deficient and KIF3B-deficient embryos (Marszalek et al. 1999; Nonaka et al. 1998; Takeda et al. 1999). These data suggest that KIF3A and KIF3B both act at an earlier step than Lefty-2 in the L/R determination pathway.

Surprising and more revealing findings were obtained by observing the nodes in KIF3A-deficient and KIF3B-deficient embryos. Although the nodal pit cells of wild-type embryos have monocilia composed of $9+0$ microtubules on the ventral surface, the cilia are either completely absent or very short and only found sporadically in the ventral nodes of embryos lacking either KIF3A or KIF3B 
(Fig. 1I). In addition, immunocytochemistry revealed that KIF3A and KIF3B are localized in the monocilia of the nodal pit cells in wildtype embryos (Nonaka et al. 1998; Takeda et al. 1999). Taken together with the results of studies on intraflagellar transport in lower eukaryotes (for reviews, see Rosenbaum and Witman 2002; Scholey 2003), these findings suggest that KIF3 is essential for ciliogenesis in nodal pit cells through the transport of protein complexes in the monocilia (Fig. 1J).

The above-described studies clarified why a lack of KIF3 leads to a loss of cilia. However, why the absence of monocilia on nodal pit cells was related to the randomness of $\mathrm{L} / \mathrm{R}$ asymmetry was still unknown. Although the monocilia in the node in wild-type embryos were thought to be immotile because of the peculiar $9+0$ arrangement of their microtubules, we reexamined the behaviors of the monocilia in both wild-type and mutant embryos in vivo using video-light microscopy.

Surprisingly, we observed that the monocilia are in fact vigorously rotating at approximately $600 \mathrm{rpm}$ (Fig. 2B,C; see online Movies 1 and 2 at http://beta.cshperspectives.cshlp. org). Most motile cilia and flagella, such as the cilia in the airway and sperm, only move back and forth and thus perform wavelike movements. However, the rotational movement of the nodal monocilia is unique. The dynein motor for propelling the ciliary movement is conserved. We observed both the inner and outer dynein arms using electron microscopy and confirmed the localizations of the axonemal outer dynein arms in the monocilia using immunofluorescence staining (Takeda et al. 1999).

It still remained puzzling why this rotational movement was related to $\mathrm{L} / \mathrm{R}$ determination. To answer this question, we sought to clarify the overall effect of this ciliary movement. We applied submicron-sized fluorescent beads as passive tracers of fluid flow in the extraembryonic fluid covering the nodal region in wildtype embryos. Quite surprisingly, the fluorescent beads tended to enter the node from the right-hand side and moved unidirectionally toward the left-hand side at a rate of $2-5 \mu \mathrm{m} / \mathrm{s}$
(Fig. 2D,E; see online Movie 1 at http:// beta.cshperspectives.cshlp.org). These observations indicate that there is leftward unidirectional flow of extraembryonic fluid at the nodal region in wild-type embryos, which we designated nodal flow. In mutant embryos lacking KIF3A or KIF3B, the fluorescent beads showed only Brownian movement (Nonaka et al. 1998; Takeda et al. 1999). These studies led us to hypothesize that the monocilia in the node are essential for $\mathrm{L} / \mathrm{R}$ determination of the human body by rotating and generating leftward nodal flow at the nodal region. We further hypothesized that a putative morphogen $\mathrm{X}$ is secreted into the extraembryonic fluid at the ventral node and forms a concentration gradient owing to the nodal flow, which subsequently turns on the switch for the expression of genes such as Lefty-2 and Nodal at the left side of the node and determines the $\mathrm{L} / \mathrm{R}$ asymmetry.

Analyses of inversus viscerum (iv) mutant mice further supported this hypothesis (Okada et al. 1999; Supp et al. 1999). iv mice are spontaneous mouse mutants that show randomization of $\mathrm{L} / \mathrm{R}$ determination (Hummel and Chapman 1959). A mouse axonemal (ciliary) dynein gene, Lrd (Left-right dynein), has been identified as the gene responsible for both iv and Lgl (Legless), which also results in $\mathrm{L} / \mathrm{R}$ randomization (Supp et al. 1997). Lrd is a member of the dynein superfamily of genes designated DLP11 (Tanaka et al. 1995) and is exclusively expressed by nodal cells at 7.5 days postcoitum (Supp et al. 1997). In embryos heterozygous for $i v$, nodal cilia rotate as rapidly as those in wild-type embryos ( $\sim 600 \mathrm{rpm}$ ) (Okada et al. 1999). This rapid movement produces a fast $(2-5 \mu \mathrm{m} / \mathrm{s})$ and smooth leftward flow of extraembryonic fluid in the ventral node. In contrast, the nodal cilia seldom move in $i v$ homozygous embryos and appear rigid (Okada et al. 1999). Furthermore, beads added to the extraembryonic fluid show only Brownian movement (Okada et al. 1999). Thus, nodal cilia in iv homozygous mice cannot rotate owing to a mutation in Lrd and consequently cannot generate the leftward nodal flow required for normal $\mathrm{L} / \mathrm{R}$ 
Left-Right Determination by Nodal Flow

determination. Furthermore, left or right isomerism (both sides of the heart adopting the left, or both the right fate) was rare both in iv and Kif3 mutant mice. This suggests that another robust mechanism(s) would exist to avoid isomerism, a severe lethal disorder because of the abnormal formation of cardiovascular system. Nodal flow, therefore, would bias this machinery to produce asymmetry in a stereotypic manner.

This nodal flow hypothesis was directly tested by culturing mouse embryos under artificial flow conditions (Nonaka et al. 2002). The laterality of the embryos was controlled by the direction of the artificial flow. Under strong rightward artificial flow, the flow in the node was reversed, as was the laterality of the embryo. These experiments showed that the direction of nodal flow determines the initial $\mathrm{L} / \mathrm{R}$ symmetry breaking. Following studies showed that the nodal flow is not specific to mouse but is widely conserved among vertebrates (Essner et al. 2005; Kramer-Zucker et al. 2005; Okada et al. 2005; Schweickert et al. 2007), and we have shown the nodal flow in rabbit embryo whose structure is closest to early human embryo (Okada et al. 2005).

After the discovery of nodal flow, two important questions remained. First, how does the rotational movement of the monocilia generate leftward unidirectional flow? Second, how does the embryo interpret the direction of the nodal flow to trigger $\mathrm{L} / \mathrm{R}$ asymmetric gene expression? We discuss these issues in the following sections.

\section{THE DIRECTION OF THE NODAL FLOW IS DETERMINED BY THE TILT OF THE ROTATION AXIS}

As mentioned above, the mechanism for determining the directionality of the nodal flow is the most fundamental mechanism for stereotyped breaking of the L/R symmetry of the human body. When we first reported the discovery of nodal flow, many hypotheses were proposed to explain the flow's directionality. A naive explanation would be that the flow is produced by unidirectional beating of the nodal cilia. However, this explanation is inconsistent with the observations. The movement of the nodal cilia is different from that of other cilia. Most cilia and flagella make flow by unidirectional power stroke, which is planar or almost planar, and in the direction of the flow (planar beating). The direction of this beating plane is considered to be determined by the alignment of the central pair microtubules, although the precise mechanism is elusive.

Nodal cilia, however, rotate helically rather than beat in a plane. This unique movement would be related to the helical symmetry of the cilia structure by the lack of the central pair of microtubules (Fig. 2A), as supported by the numerical simulation studies (Brokaw 2005). Thus, a specific mechanism is required to produce unidirectional leftward flow from the rotating cilia because the simple circling movement of the nodal cilia only produces a vortex in the node.

Initially, the triangular shape of the node and the arrangement of the nodal cilia were proposed to be involved in generating the leftward flow (Nonaka et al. 1998; Okada et al. 1999; Vogan and Tabin 1999). This idea was rejected based on both observations and simulation studies. To test this idea, we observed the nodal flow in other vertebrates, such as fish and rabbits (Okada et al. 2005). The shapes of their nodes are round or elliptic and differ from that of the mouse node. However, unidirectional leftward nodal flow is still produced in these nodes by the rotating nodal cilia.

We, then, focused on the movement of the nodal cilia, because the movement of the cilia themselves or the coordination of the movement among adjacent cilia may determine the directionality. We traced the cilia's movement with a high-speed camera at 500 frames/s and determined their trajectories in three dimensions (Okada et al. 2005). As observed in previous low-time-resolution studies, the nodal cilia showed a clockwise rotational-like motion (Fig. 2C; see online Movie 3 at http:// beta.cshperspectives.cshlp.org). The axis did not show a lateral bias from the midline but tilted by $40 \pm 10^{\circ}$ to the posterior from the vertical angle (Fig. 3A,B). This posterior tilt of 
N. Hirokawa, Y. Tanaka, and Y. Okada

A
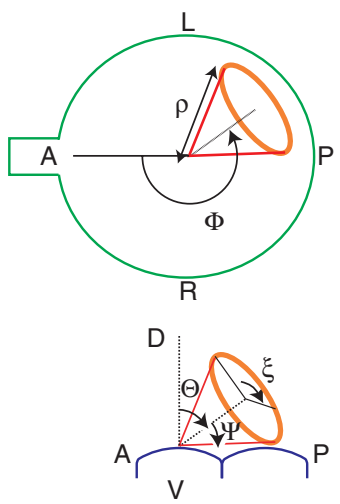

B

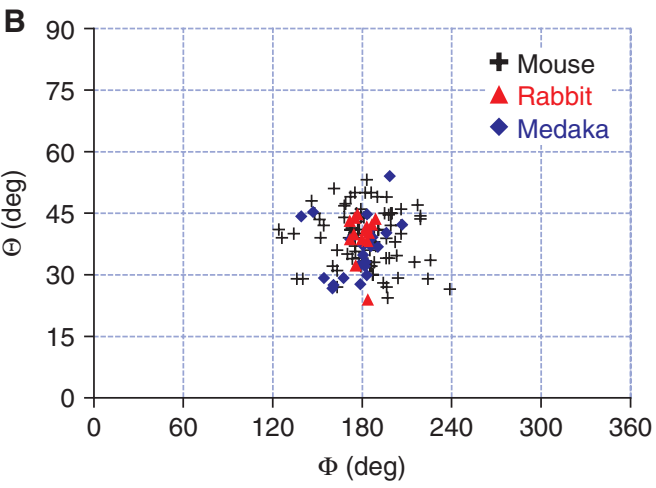

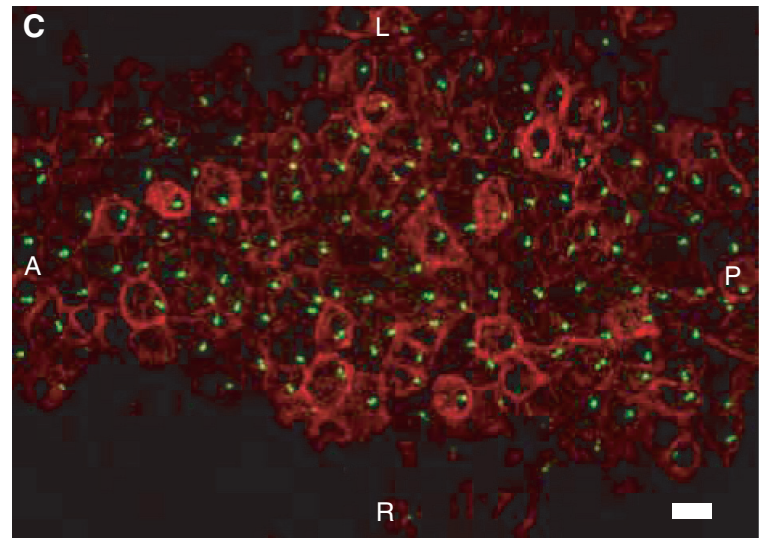
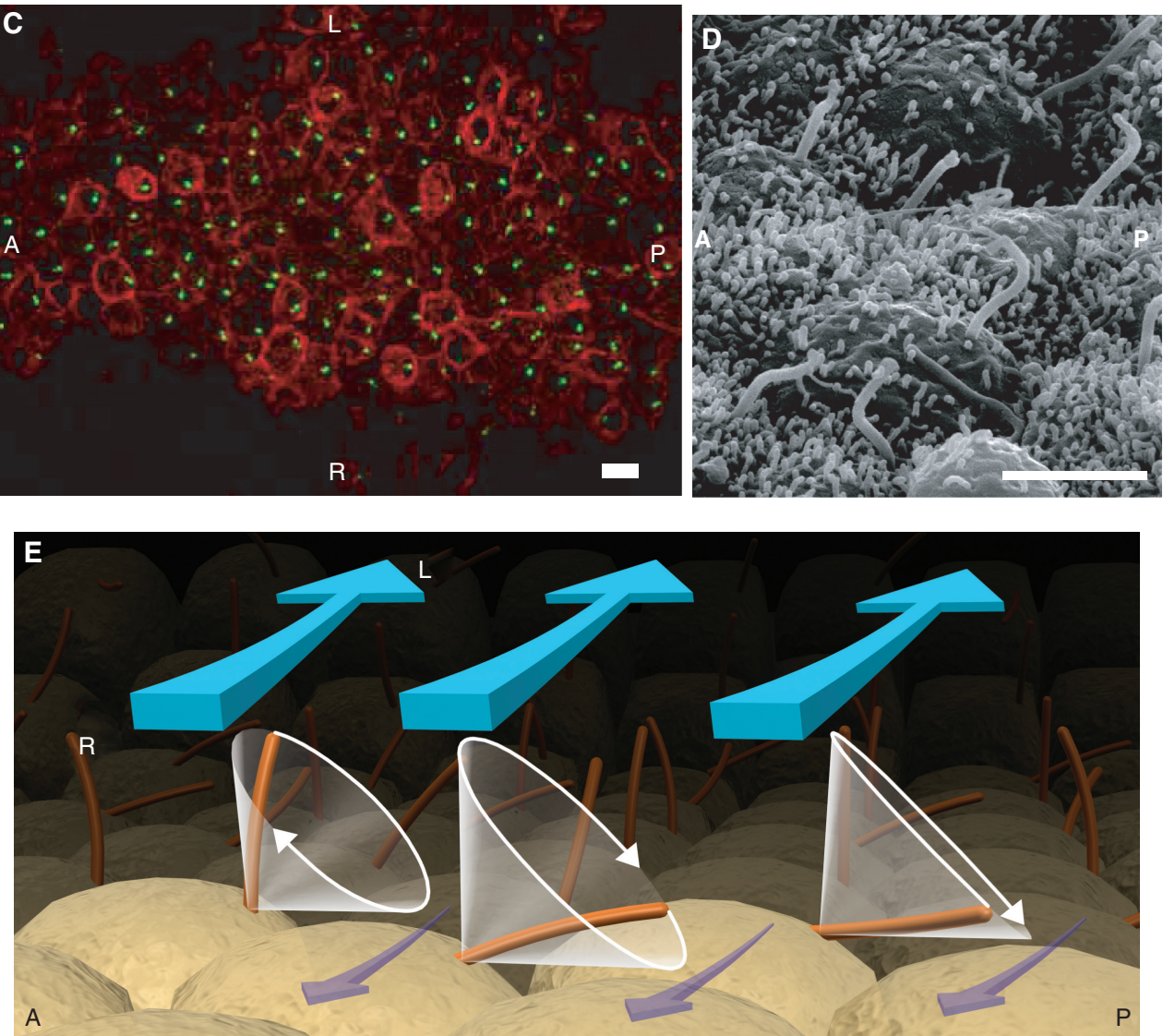

Figure 3. $(A, B)$ Posteriorly tilted rotation of nodal cilia. (A) Definition of the analysis parameters. The conic rotation of the nodal cilium was parameterized with the following parameters: tilt of axis, $\Theta$; direction of the axis, $\Phi$; slant height, $\rho$; and apex angle, $\tilde{\Psi}$. (B) Distributions of the parameters in mouse, rabbit, and medaka embryos. $(C, D)$ Posterior bias in the positions of the roots of the nodal cilia. (C) Fluorescent micrograph of a rabbit node. Green dots show the roots of the nodal cilia, whereas red staining shows the cells' boundaries. The orientation is indicated in the panels as anterior (A), posterior (P), left $(\mathrm{L})$, and right $(\mathrm{R})$. Most of the green dots are located on the posterior side. Scale bar $=10 \mu \mathrm{m}$. $(D)$ High-magnification scanning electron micrograph of rabbit nodal ciliated cells. Note the domelike curvature of the apical plasma membrane and posteriorly tilted projection of the monocilia nearly perpendicular to the plasma membrane. Scale bar $=5 \mu \mathrm{m}$. (Continued) 
the rotation axis was conserved among all vertebrates examined (fish, mice, and rabbits) (Okada et al. 1999, 2005). Nonaka et al. (2005) experimentally supported this idea. They reproduced the tilted rotation and directional flow by making a model of the array of the nodal cilia with copper wire and a stepping motor.

The Cartwright et al. (2004) numerical simulation study also suggested that the triangular array of vertically aligned local vortices could only produce general circulation near the edge. Their study also suggested that the array of posteriorly tilted cilia can produce leftward flow above the cilia, but it is accompanied by an equally fast rightward flow below the cilia. This is inconsistent with observations because rightward flow is not observed below the cilia. Instead, the rightward counterflow is observed in the top half of the node cavity in mouse embryos (see online Movie 4 at http://beta. cshperspectives.cshlp.org) (Okada et al. 2005). This is a slow passive flow at the top to balance the leftward nodal flow at the bottom of the cavity.

We proposed that the viscosity of the fluid and the shear resistance of the node's stationary surface would slow down the rightward current near the surface (Okada et al. 2005). As observed by our group and others (Nonaka et al. 2005), the cilia rotate conically with a tilt angle of approximately $40^{\circ}$ and a semicone angle of approximately $50^{\circ}$ (Fig. 3B). Consequently, the cilia make a leftward swing toward the fluid and a rightward sweep toward the surface. Because nodal flow is in the low Reynolds-number regime (with a magnitude of $10^{-3}$ ), viscous drag or shear resistance from the stationary cell surface is not negligible. The stationary surface would retard the movement of fluids by shear resistance. Thus, the rightward sweep is less effective than the leftward sweep in generating fluid movement (Fig. 3E). Numerical simulation studies clarified that the effect of the surface, or shear resistance, is the major factor for converting the posteriorly tilted rotation of the cilia into the unidirectional leftward flow (Buceta et al. 2005; Cartwright et al. 2007; Smith et al. 2007, 2008). They also suggested that that the tilt angle of the nodal cilia may be optimized to produce fast unidirectional flow.

The mechanism of the de novo generation of the leftward flow has thus been clarified by the combination of the experimental and theoretical studies. As proposed by Brown and Wolpert (1990), the stereotyped breakage of $\mathrm{L} / \mathrm{R}$ symmetry is enabled by a chiral structure aligned with respect to the AP and DV axes. Cilia in the ventral node tilted toward the posterior represent exactly that kind of structure. The structural properties of the nodal cilia determine the flow's direction without relying on any a priori L/R asymmetry (Fig. 3E). The clockwise rotation of the cilia reflects the chiral architecture of the nodal monocilia, in which the dynein arms are aligned in a clockwise manner on the side of each doublet microtubule (Fig. 2A) (for the modeling study, see Brokaw 2005). The nodal cilia are ventral protrusions from the nodal pit cells. Interestingly, the basal bodies or roots of the monocilia, are positioned at the cell posterior, possibly reflecting the planar cell polarity (PCP) of the nodal pit cells toward the AP axis (Fig. 3C,D) (Okada et al. 2005; see Vladar et al. 2009). The convex curvature of the nodal pit cells may explain how this posterior localization contributes to the tilting of the rotation axes (Fig. 3D). However, the precise molecular nature of this PCP pathway is still open to future research (Okada et al. 2005; Aw and Levin 2009).

Figure 3. (Continued). (E) Model of the generation of nodal flow. The orientation is indicated as anterior (A), posterior $(\mathrm{P})$, left $(\mathrm{L})$, and right $(\mathrm{R})$. The conic rotation of the nodal cilia is posteriorly tilted. Therefore, the cilia move in a nearly perpendicular manner from right to left. The return movement from left to right occurs just above the cell surface. The viscous drag thus dumps the movement of the fluid to the right (purple). As a result, unidirectional leftward flow (blue) is generated. (Figure modified from Okada et al. 2005 and Hirokawa et al. 2006 with permission.) 
N. Hirokawa, Y. Tanaka, and Y. Okada

\section{TWO MODELS FOR GENERATING LEFT-RIGHT ASYMMETRIC GENE EXPRESSION BY NODAL FLOW}

The next important question is how the cells in the embryo interpret leftward nodal flow. Other developmental processes, especially body-axis determination in invertebrate animals such as the fruit fly Drosophila, use concentration gradients of diffusible secreted chemicals such as proteins or lipids as positional information along the body axis, and these chemicals are referred to as morphogens (Gurdon and Bourillot 2001). Based on this analogy, it was first proposed that nodal flow may produce a concentration gradient of a morphogen in the cavity of the node (Fig. 4A) (Nonaka et al. 1998; Okada et al. 1999).

Although the chemical gradient model is simple, it is associated with great difficulty. It is questionable whether nodal flow can actually generate a concentration gradient of a secreted chemical morphogen in a closed cavity of node, where the leftward flow is balanced by the rightward counterflow (Cartwright et al. 2004, 2007). A potential consequence of this counterflow may be that secreted molecules are uniformly distributed. Thus, we examined this model experimentally (Okada et al. 2005). We introduced a caged-fluorescently labeled protein into the nodal cavity and locally activated it using focused ultraviolet irradiation to simulate continuous secretion. Interestingly, only proteins of $20-40 \mathrm{kDa}$ generated stationary concentration gradients. These sizes match those of candidate proteinaceous morphogens such as fibroblast growth factor (FGF) 8 and Nodal. This size dependency was explained by a difference in the flow rates of the leftward nodal flow and rightward counterflow (Buceta et al. 2005; Okada et al. 2005). For larger proteins, advection dominates over diffusion, and they circulate in the cavity. For smaller proteins, diffusion dominates over advection, and they rapidly diffuse symmetrically in the cavity. For medium-sized proteins of approximately $20 \mathrm{kDa}$, the diffusion coefficient is approximately $1 \times 10^{-6} \mathrm{~cm}^{2} / \mathrm{s}$. The half-width of the node is approximately $50 \mu \mathrm{m}$. The flow rates of the leftward nodal flow and rightward counterflow are approximately 3 and $1 \mu \mathrm{m} / \mathrm{s}$, respectively. Thus, the Peclet numbers, dimensionless number relating the rate of advection of a flow to its rate of diffusion, for the nodal flow and counterflow are approximately 1.5 and 0.5 , respectively. Hence, advection by the leftward flow dominates over diffusion, whereas diffusion dominates over advection by the rightward flow, such that medium-sized proteins accumulate on the left side. Local transient ultraviolet irradiation experiments confirmed this theoretical explanation (Okada et al. 2005). Interestingly, the Peclet number was conserved in rabbits, despite the species differences in the size and shape of the ventral node, and the velocity of the nodal flow. These results suggest that some protein molecules may spontaneously generate a chemical gradient in the nodal cavity.

As a critique of the chemical gradient model, an alternative model was proposed that assumes that the flow itself physically invokes cellular responses. This model was reinforced by the finding that some nodal cilia, especially those in the peripheral region of the ventral node, are immotile (McGrath et al. 2003). Nodal flow establishment is immediately followed by elevation of the intracellular calcium concentration on the left edge of the node (Fig. 4D). Thus, it has been suggested that the immotile cilia on the edge of the node serve as mechanical flow sensors and trigger a calcium response depending on the flow's direction (Fig. 4B). Hence, this physical stimulation model is now often termed the two-cilia hypothesis, referring to one type of cilia for flow generation and a second type of cilia for flow sensing (Tabin and Vogan 2003). This proposal gathered interest from the relation to polycystic kidney disease, in which loss of flow sensing by the primary cilia is considered to lead to cyst formation (Nauli and Zhou 2004). However, our experiments excluded this model, and suggested that an unexpected novel mechanism produces $\mathrm{L} / \mathrm{R}$ gradient(s) of morphogen(s). 
A

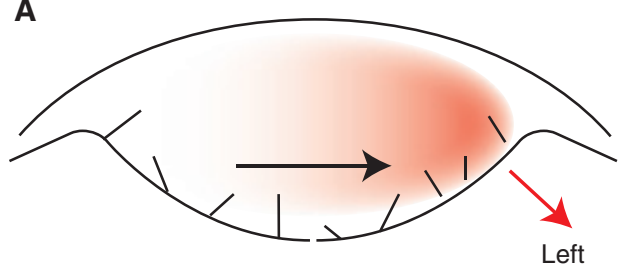

B

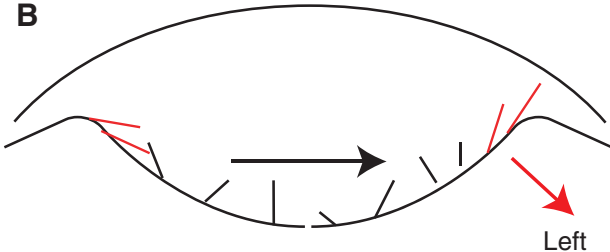

Left

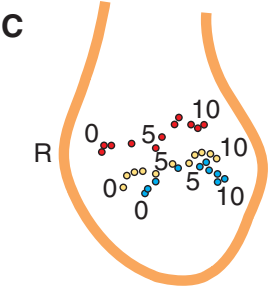

DMSO

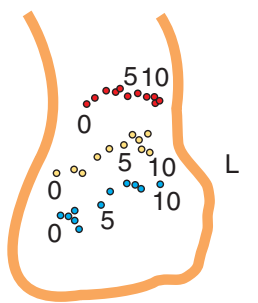

SU5402

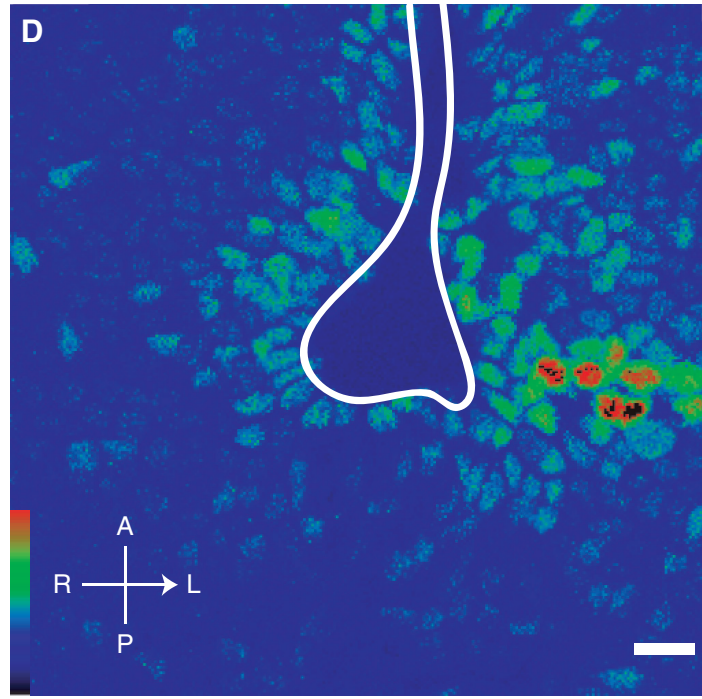

E
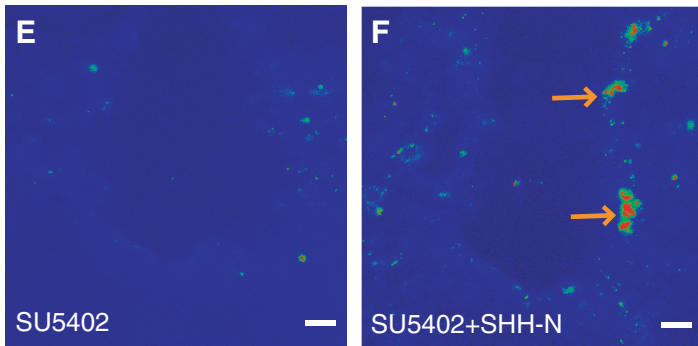

Figure 4. Fibroblast growth factor (FGF) signaling is essential for left-specific calcium elevation but not for the nodal flow. ( $A$ and $B$ ) Models for the mechanism of interpretation of the nodal flow to the downstream signaling. (A) Concentration gradient model. Left-right asymmetry of the concentration of morphogen(s) in the consequence of nodal flow (black arrow) stimulates the cells on the left edge of the node, which further propagate the signal to the downstream (red arrow). (B) Two-cilia model. Cilia on the edge are specifically differentiated to sensing the flow, and those on the left edge, but not on the right edge, are activated by the leftward nodal flow to activate the signaling cascade. $(C)$ Leftward fluid flow is not affected by SU5402 treatment. Camera lucida of three traces of fluorescent beads for DMSO (carrier)- or SU5402-treated embryos at the 1-3 somite stage, plotted every 1 second for 10 seconds. Upper, anterior; lower, posterior. Scale bar, $10 \mu \mathrm{m}$. $(D-F)$ FGF regulates left-specific $\mathrm{Ca}^{2+}$ elevation. Ventral view of $\mathrm{Ca}^{2+}$ imaging of wild type embryos, pharmacologically treated as indicated, and represented by pseudocolor ( signal intensity: red $>$ green $>$ blue $>$ black). Note that FGF inhibitor eliminates the left-specific $\mathrm{Ca}^{2+}$ elevation, and that addition of SHH-N peptide could replenish the $\mathrm{Ca}^{2+}$ elevation in cells on the left edge of the node (F, arrows). Upper, anterior; lower, posterior. Scale bars, $20 \mu \mathrm{m}$. (Figure modified from Tanaka et al. 2005 and Hirokawa et al. 2009 with permission.)

\section{IDENTIFICATION OF NODAL \\ VESICULAR PARCELS, A NOVEL FORM OF TRANSPORT MACHINERY FOR \\ SIGNALING MOLECULES}

We re-examined the signaling processes in the node using pharmacological perturbations (Tanaka et al. 2005). We first identified FGF receptors localized to the cilia and cell surface of the node cells (Tanaka et al. 2005). Thus, these cilia are suggested to have dual functions: motility and sensation. Then, we applied specific inhibitors of FGF receptors to the mouse embryos. In consequence, they significantly suppressed the calcium response on the left edge of the node (Fig. 4E), whereas the leftward nodal flow remained unaffected (Fig. 4C). 
Thus, the calcium elevation is dependent not only on the flow but also on growth factor signaling. This blockage was reversed by the addition of other signaling molecules, namely sonic hedgehog (SHH) or retinoic acid (RA) (Fig. 4F). These results strongly argue against the two-cilia hypothesis. The calcium response is not directly triggered by the mechanical stimuli of the flow itself, but is mediated by chemical signaling molecules. Therefore, how could the biochemical reactions activate cells on the left side of the node?

We then observed the dynamics of the membrane lipid in the ventral node by using fluorescent lipophilic dye DiI. Quite intriguingly, we identified extracellular materials that are secreted from the node surface and transported to the left, corresponding to the stage of the nodal flow (Fig. 5A; see online Movie 5 at http://beta.cshperspectives.cshlp.org) (Tanaka et al. 2005). We termed these materials nodal vesicular parcels (NVPs) and further characterized them using light and electron microscopy, as well as pharmacological perturbations.

NVPs appear to be secreted from the surface of the node by an initial launch in random directions, probably driven by actin-mediated extension of a special form of long dynamic microvilli (Fig. 5D,E; see online Movie 6 at http://beta.cshperspectives.cshlp.org), followed by transport to the left side by the ciliamediated nodal flow. Many spherical NVP precursor candidates were present on the node's surface (Fig. 5B). Electron microscopic observations suggested that NVPs are membranesheathed lipoprotein particles that are often associated with microvilli (Fig. 5B,C,D). According to light microscopic observations, these microvilli appeared to elongate in 10 seconds to launch the NVPs from their original sites, although the NVPs sometimes crawl along the surface. The launch of NVPs is dependent on FGF signaling, because a specific inhibitor of FGF receptor (FGFR), SU5402, and a dominant-negative FGFR polypeptide can similarly inhibit their launching from the surface. The launch of NVPs does not seem to be dependent on cilia because NVPs are observed in the nodes of KIF3 knockout embryos deficient in cilia formation. The negative effects of FGFR inhibition can be reversed by adding a recombinant polypeptide of the amino-terminal half of SHH (SHH-N) or RA to the culture medium, similar to the case of the calcium elevation. According to spatiotemporal order, NVP signals are probably the trigger of the calcium elevation on the left side of the node.

Furthermore, SHH and RA were specifically detected by immunofluorescence microscopy and immunoelectron microscopy at the possible releasing sites. Thus, the NVP release would be enhanced by extrinsic application of cargo molecules of the NVPs. This suggested that the accumulation of either SHH or RA at the releasing sites may locally enhance the NVP launch downstream of FGF signaling (Tanaka et al. 2005). The primary source of SHH is probably the nodal pit cells according to a previous report (Echelard et al. 1993). The action of RA in the node does not seem to involve conventional nuclear receptormediated signaling (Sirbu and Duester 2006), suggesting the existence of a new reception mechanism of RA and SHH on the cell surface to locally activate NVP release. RA and SHH may synergistically share activity of enhancing NVP release, because Shh knockout mouse embryos can still release NVPs (Y. Tanaka and N. Hirokawa, unpublished observations), and knockout for RA-synthesizing Raldh2 gene did not modify the left-right randomization phenotype in iv/iv mice (Vermot et al. 2005). However, RA is certainly described to act upstream of the expression of the Lefty-2, Nodal, and Pitx2 genes in the left lateral plate mesoderm, because treatment of embryos either with RA or its inhibitors can significantly change the expression patterns of these leftspecific genes and heart morphogenesis (Tsukui et al. 1999; Chazaud et al. 1999; Wasiak and Lohnes, 1999). SHH is also essential for expressing Lefty-1 in prospective floor plate ( pfp) cells on the left side of the midline (Tsukui et al. 1999). Thus, these molecules are probably cooperatively or coordinately acting on NVP signaling to play roles in left-right determination. Hedgehog proteins have also been shown to be secreted from the cell surface 

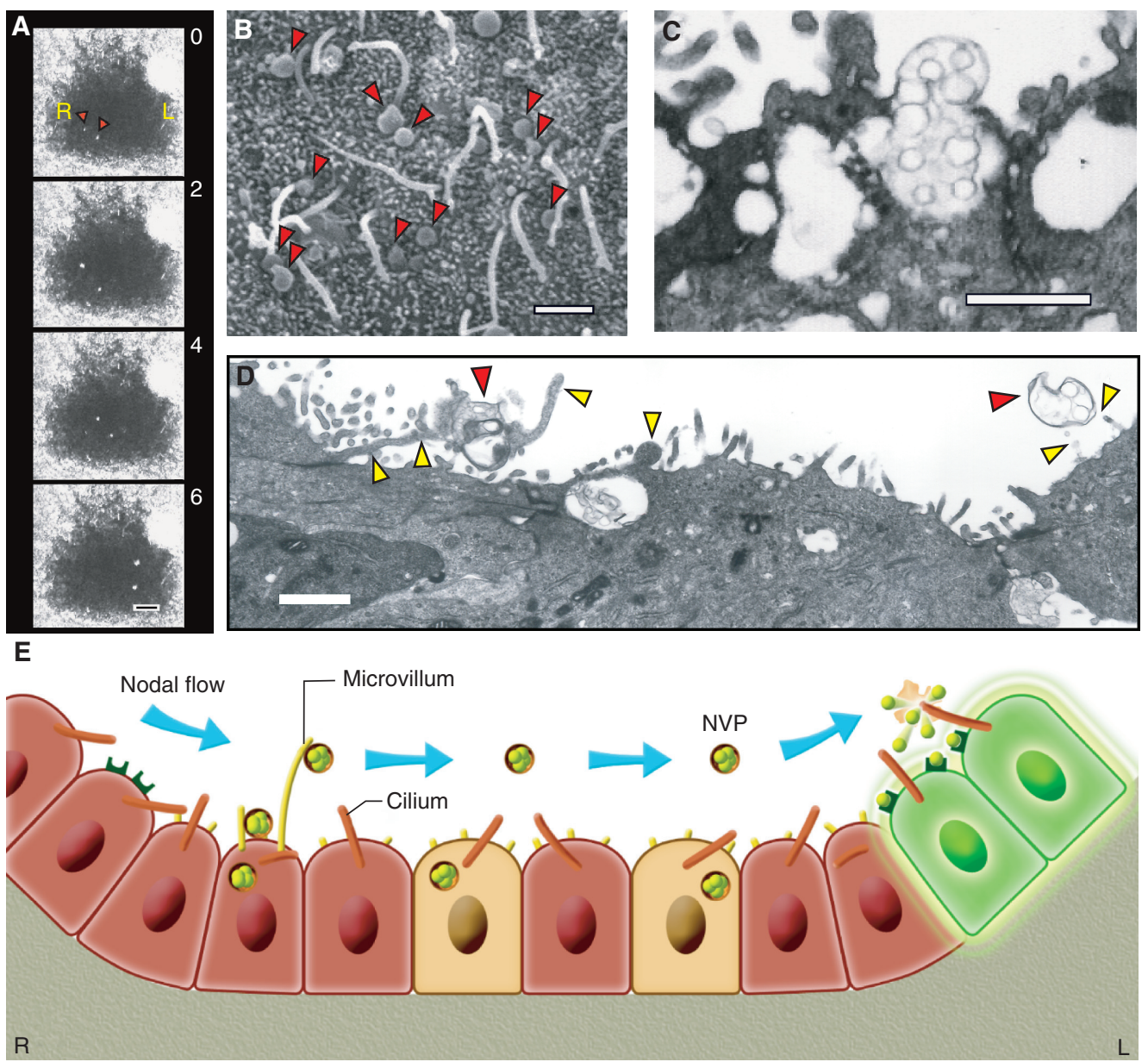

Figure 5. (A) Time-lapse optical microscope images of flowing nodal vesicular parcels (NVPs). The orientation is indicated in the panel as left (L) and right (R). NVPs (arrowheads) are transferred to the left side by the nodal flow (see online Movie 5 at http://beta.cshperspectives.cshlp.org). Scale bar $=10 \mu \mathrm{m}$. (B) Scanning electron micrograph of the ventral surface of nodal pit cells. Red arrowheads indicate NVP precursors. Scale bar $=2 \mu \mathrm{m}$. $(C, D)$ Transmission electron micrographs of nodal pit cells. $(C)$ Transition state of NVP release in an SU5402-treated embryo. Scale bar $=1 \mu \mathrm{m}$. (D) NVPs (red arrowheads) are associated with microvilli (yellow arrowheads). Scale bar $=1 \mu \mathrm{m}$. (E) Schematic representation of NVP flow. The orientation is indicated in the panel as left (L) and right (R). NVPs appear to be released from dynamic microvilli (see online Movie 6 at http://beta.cshperspectives.cshlp.org), transported to the left side by the nodal flow (see online Movie 5 at http://beta.cshperspectives.cshlp.org), and fragmented with the aid of cilia at the left periphery of the node (see online Movie 7 at http://beta.cshperspectives.cshlp.org). The green halos indicate calcium elevation as a sign of cell activation on the left side of the node. (Figure modified from Tanaka et al. 2005 with permission.)

by membrane-sheathed extracellular particles (Satir and Christensen 2008; Kiprilov et al. 2008). Thus, NVPs are proposed to be a good model system for elucidating the biological processes involving SHH/RA signaling, which could contribute in resolving many other signaling mechanisms.
The released NVPs are transferred to the left side by the nodal flow (Fig. 5A; see online Movie 5 at http://beta.cshperspectives.cshlp. org). Interestingly, most NVPs appeared to be fragmented on the left side and absorbed by the nodal cell surface (see online Movie 7 at http://beta.cshperspectives.cshlp.org). This 
mechanism ensures that the NVPs are unidirectionally transferred to the left side (Fig. 5E), without circulating in the node forever, and that the contents of the NVPs, probably SHH, RA, lipoproteins, and other unidentified morphogens, create the concentration gradient. It is still puzzling how the membrane sheaths of NVPs are ruptured and how the NVPs are fragmented at the proximity of the left wall. This fragmentation probably involves both fluid mechanical and biochemical processes, and its nature is still being investigated.

In summary, these results collectively suggest that the $\mathrm{L} / \mathrm{R}$ asymmetric development would occur in the following manner (see online Movie 8 at http://beta.cshperspectives. cshlp.org): (1) Tilted cilia rotation produces leftward nodal flow. (2) FGF facilitates the accumulation of $\mathrm{SHH}$ and RA on NVP releasing sites on the nodal pit cell. (3) Accumulation of $\mathrm{SHH}$ and/or RA triggers NVP release from the cell surface. (4) Leftward nodal flow transports NVPs to the left edge of the node. (5) NVP is fragmented to deposit its contents at the left edge of the node. (6) Calcium elevation starts on the left edge of the node (nodal crown cells), and propagates through adjacent cells to the left lateral plate mesoderm (probably involving FGF and Notch signaling). (7) Left-specific genes start to be expressed in the left lateral plate mesoderm. There must be many loops and feedbacks in addition to this sequence, but the main stream can be summarized like this. The molecular details of this pathway are still being investigated, and the mechanisms involving both fluid dynamics and new cell biological phenomena will be key elements toward elucidating the mechanism of $\mathrm{L} / \mathrm{R}$ determination.

\section{CONCLUSION}

The long-lasting enigma of $\mathrm{L} / \mathrm{R}$ determination of the human body has been solved by the integration of interdisciplinary approaches, including biology and physics. Chiral mechanical elements, nodal cilia, are produced on the posterior-ventral surface of nodal pit cells, possibly by anterior-posterior planar cell polarity of nodal pit cells. Clockwise conic rotation of the nodal cilia generates leftward fluid flow in the nodal cavity through hydrodynamic mechanisms. This flow carries NVPs that contain signaling molecules to the left edge of the node. In this region, the NVPs are somehow fragmented to deliver their internal signaling molecules through a presently unidentified mechanism. After the fragmentation, the NVPs do not return to the right side via the counterflow in the cavity, such that vectorial transport to the left side is guaranteed.

Such a combination of mechanical processes and chemical signaling was totally unexpected. Because morphogens are generally considered to diffuse passively, the reactiondiffusion model is widely used to model the pattern formation in developmental processes (Meinhardt 1982). Nodal flow is the first evidence that advection can be a key determinant in developmental processes, and it is unlikely to be unique to $\mathrm{L} / \mathrm{R}$ determination. Following the discovery of nodal flow, developmental biologists have postulated that other developmental processes may also be mediated by the transport of signaling molecules via similar fluid flows. Recently, an axis in the brain was suggested to be dependent on the direction of the flow of cerebrospinal fluid (Sawamoto et al. 2006). Future interdisciplinary studies, such as those for $\mathrm{L} / \mathrm{R}$ determination, will further identify the fluid-mediated signaling processes essential for the human body and will deepen our understanding of the underlying biology and physics.

\section{ACKNOWLEDGMENTS}

We thank members of the Hirokawa lab for assistance and discussion. This work was supported by the Ministry of Education, Culture, Sports, Science and Technology of Japan, Grant-in-Aid for Specially Promoted Research to N.H.

\section{REFERENCES}

Afzelius BA. 1976. A human syndrome caused by immotile cilia. Science 193: 317-319. 
Aizawa H, Sekine Y, Takemura Z, Zhang Z, Nangaku M, Hirokawa N. 1992. Kinesin family in murine central nervous system. J Cell Biol 119: 1287-1296.

Alarcon VB, Marikawa Y. 2003. Deviation of the blastocyst axis from the first cleavage plane does not affect the quality of mouse postimplantation development. Biol Reprod 69: 1208-1212.

Aw S, Levin M. 2009. Is left-right asymmetry a form of planar cell polarity? Development 136: 355-366.

Beddington RSP, Robertson EJ. 1999. Axis development and early asymmetry of mammals. Cell 96: 195-209.

Bellomo D, Lander A, Harragan I, Brown NA. 1996. Cell proliferation in mammalian gastrulation: The ventral node and notochord are relatively quiescent. Dev Dyn 205: 471-485.

Brokaw CJ. 2005. Computer simulation of flagellar movement IX. Oscillation and symmetry breaking in a model for short flagella and nodal cilia. Cell Motil Cytoskelet 60: 35-47.

Brown NA, Wolpert L. 1990. The development of handedness in left-right asymmetry. Development 109: 1-9.

Buceta J, Ibañes M, Rasskin-Gutman D, Okada Y, Hirokawa N, Izpisúa-Belmonte JC. 2005. Nodal cilia dynamics and the specification of the left-right axis in early vertebrate embryo development. Biophys J 89: 2199-2209.

Capdevila J, Vogan KJ, Tabin CJ, Izpisua-Belmonte JC. 2000. Mechanisms of left-right determination in vertebrates. Cell 101: 9-21.

Cartwright JH, Piro O, Tuval I. 2004. Fluid-dynamical basis of the embryonic development of left-right asymmetry in vertebrates. Proc Natl Acad Sci 101: 7234-7239.

Cartwright JH, Piro N, Piro O, Tuval I. 2007. Embryonic nodal flow and the dynamics of nodal vesicular parcels. $J$ R Soc Interface 4: 49-55.

Chazaud C, Chambon P, Dollé P. 1999. Retinoic acid is required in the mouse embryo for left-right asymmetry determination and heart morphogenesis. Development 126: $2589-2596$.

Echelard Y, Epstein DJ, St-Jacques B, Shen L, Mohler J, et al. 1993. Sonic hedgehog, a member of a family of putative signaling molecules, is implicated in the regulation of CNS polarity. Cell 75: 1417-1430.

Essner JJ, Amack JD, Nyholm MK, Harris EB, Yost HJ. 2005. Kupffer's vesicle is a ciliated organ of asymmetry in the zebrafish embryo that initiates left-right development of the brain, heart and gut. Development 132: 1247-1260.

Gilbert SF. 2003. Developmental biology. 7th Edition. Sinauer Associates Inc, Sunderland, Massachusetts.

Gurdon JB, Bourillot PY. 2001. Morphogen gradient interpretation. Nature 413: 797-803.

Hamada H. 2002. Establishment of vertebrate left-right asymmetry. Nature Rev Genet 3: 103-113.

Harvey RP. 1998. Links in the left/right axial pathway. Cell 94: $273-276$.

Hirokawa N. 1998. Kinesin and dynein superfamily proteins and the mechanism of organelle transport. Science 279: 519-526.

Hirokawa N, Noda Y. 2008. Intracellular transport and kinesin superfamily proteins. KIFs: Structure, function and dynamics. Physiol Rev 88: 1089-1118.
Hirokawa N, Takemura R. 2005. Molecular motors and mechanisms of directional transport in neurons. Nat Rev Neurosci 6: 201-214.

Hirokawa N, Tanaka Y, Okada Y, Takeda S. 2006. Nodal flow and the generation of left-right asymmetry. Cell 125: $33-45$.

Hirokawa N, Okada Y, Tanaka Y. 2009. Fluid dynamic mechanism responsible for breaking the left-right symmetry of the human body: The nodal flow. Ann Rev Fluid Mech 41: 53-72.

Hummel KP, Chapman DB. 1959. Visceral inversion and associated anomalies in the mouse. J Hered 50: 9-13.

Kartagener M. 1933. Zur pathogenese der bronchiectasien. I Mitteilung: Bronchiectasien bei situs viscerum inversus. Betr Klin Tuberk 83: 498-501.

Kaufman MH. 1992. The atlas of mouse development. 1st ed. Academic Press, San Diego, California.

Kiprilov EN, Awan A, Desprat R, Velho M, Clement CA, Byskov AG, Andersen CY, Satir P, Bouhassira EE, Christensen ST, et al. 2008. Human embryonic stem cells in culture possess primary cilia with hedgehog signaling machinery. J Cell Biol 180: 897-904.

Kramer-Zucker AG, Olale F, Haycraft CJ, Yoder BK, Schier AF, Drummond IA. 2005. Cilia-driven fluid flow in the zebrafish pronephros, brain and Kupffer's vesicle is required for normal organogenesis. Development 132: 1907-1921.

Levin M. 2005. Left-right asymmetry in embryonic development: A comprehensive review. Mech Dev 122: 3-25.

Marszalek JR, Ruiz-Lozano P, Roberts E, Chien KR, Goldstein LSB. 1999. Situs inversus and embryonic ciliary morphogenesis defects in mouse mutants lacking the KIF3A subunit of kinesin-II. Proc Nat Acad Sci 96: 5043-5048.

McGrath J, Somlo S, Makova S, Tian X, Brueckner M. 2003. Two populations of node monocilia initiate left-right asymmetry in the mouse. Cell 114: 61-73.

Meinhardt H. 1982. Models of biological pattern formation. London, Academic.

Miki H, Setou M, Kaneshiro K, Hirokawa N. 2001. All kinesin superfamily protein, KIF, genes in mouse and human. Proc Natl Acad Sci 98: 7004-7011.

Miki H, Okada Y, Hirokawa N. 2005. Analysis of the kinesin superfamily: insights into structure and function. Trends Cell Biol 15: 467-476.

Nauli SM, Zhou J. 2004. Polycystins and mechanosensation in renal and nodal cilia. BioEssays 26: 844-856.

Nonaka S, Tanaka Y, Okada Y, Takeda S, Harada A, et al. 1998. Randomization of left-right asymmetry due to loss of nodal cilia generating leftward flow of extraembryonic fluid in mice lacking KIF3B motor protein. Cell 95: 829-837.

Nonaka S, Shiratori H, Saijoh Y, Hamada H. 2002. Determination of left-right patterning of the mouse embryo by artificial nodal flow. Nature 418: 96-99.

Nonaka S, Yoshiba S, Watanabe D, Ikeuchi S, Goto T, et al. 2005. De novo formation of left-right asymmetry by posterior tilt of nodal cilia. PLoS Biol 3: pe268.

Okada Y, Nonaka S, Tanaka Y, Saijoh Y, Hamada H, Hirokawa N. 1999. Abnormal nodal flow precedes situs inversus in iv and inv mutant mice. Mol Cell 4: 459-468. 
N. Hirokawa, Y. Tanaka, and Y. Okada

Okada Y, Takeda S, Tanaka Y, Izpisúa-Belmonte JC, Hirokawa N. 2005. Mechanism of nodal flow: A conserved symmetry breaking event in left-right axis determination. Cell 121: 633-644.

Rosenbaum JL, Witman GB. 2002. Intraflagellar transport. Nat Rev Mol Cell Biol 3: 813-825.

Satir P, Christensen ST. 2007. Overview of structure and function of mammalian cilia. Annu Rev Physiol 69: $377-400$.

Satir P, Christensen ST. 2008. Structure and function of mammalian cilia. Histochem Cell Biol 129: 687-693.

Sawamoto K, Wichterle H, Gonzalez-Perez O, Cholfin JA, Yamada M, et al. 2006. New neurons follow the flow of cerebrospinal fluid in the adult brain. Science 311: 629-632.

Scholey JM. 2003. Intraflagellar transport. Annu Rev Cell Dev Biol 19: 423-443.

Schweickert A, Weber T, Beyer T, Vick P, Bogusch S, Feistel K, Blum M. 2007. Cilia-driven leftward flow determines laterality in Xenopus. Curr Biol 17: 1760-1766.

Sirbu IO, Duester G. 2006. Retinoic-acid signalling in node ectoderm and posterior neural plate directs left-right patterning of somitic mesoderm. Nat Cell Biol 8: 271-277.

Smith DJ, Gaffney EA, Blake JR. 2007. Discrete cilia modeling with singularity distributions: Application to the embryonic node and the airway surface liquid. Bull Math Biol 69: 1477-1510.

Smith DJ, Blake JR, Gaffney EA. 2008. Fluid mechanics of nodal flow due to embryonic primary cilia. $J R S o c$ Interface 5: 567-573.

Supp DM, Brueckner M, Kuehn MR, Witte DP, Lowe LA, et al. 1999. Targeted deletion of the ATP binding domain of left-right dynein confirms its role in specifying development of left-right asymmetries. Development 126: 5495-5504.

Supp DM, Witte DP, Potter SS, Brueckner M. 1997. Mutation of an axonemal dynein affects left-right asymmetry in inversus viscerum mice. Nature 389: 963-966.

Tabin CJ, Vogan KJ. 2003. A two-cilia model for vertebrate left-right axis specification. Genes Dev 17: 1-6.

Tahirovic S, Bradke F. 2009. Neuronal polarity. Cold Spring Harb Perspect Biol 1: a001644.

Takeda S, Yonekawa Y, Tanaka Y, Okada Y, Nonaka S, Hirokawa N. 1999. Left-right asymmetry and kinesin superfamily protein KIF3A: New insights in determination of laterality and mesoderm induction by kif3A ${ }^{-/-}$mice analysis. J Cell Biol 145: 825-836.

Tanaka Y, Zhang Z, Hirokawa N. 1995. Identification and molecular evolution of new dynein-like protein sequences in rat brain. J Cell Sci 108: 1883-1893.

Tanaka Y, Okada Y, Hirokawa N. 2005. FGF-induced vesicular release of Sonic hedgehog and retinoic acid in leftward nodal flow is critical for left-right determination. Nature 435: $172-177$.

Tsukui T, Capdevila J, Tamura K, Ruiz-Lozano P, Rodríguez-Esteban C, Yonei-Tamura S, Magallón J, Chandraratna RAS, Chien K, Blumberg B, et al. 1999. Multiple left-right asymmetry defects in $\mathrm{Shh}^{-/-}$ mutant mice unveil a convergence of the Shh and retinoic acid pathways in the control of Lefty-1. Proc Natl Acad Sci 96: 11376-11381.

Vermot J, Llamas JG, Fraulob V, Niederreither K, Chambon P, Dollé P. 2005. Retinoic acid controls the bilateral symmetry of somite formation in the mouse embryo. Science 308: $563-566$.

Vladar EK, Antic D, Axelrod JD. 2009. Planar cell polarity signaling: the developing cell's compass. Cold Spring Harb Perspect Biol 1: a002964.

Vogan KJ, Tabin CJ. 1999. A new spin on handed asymmetry. Nature 397: 297-298.

Wasiak S, Lohnes D. 1999. Retinoic acid affects left-right patterning. Dev Biol 215: 332-342.

Yost HJ. 1999. Diverse initiation in a conserved left-right pathway? Curr Opin Genetics and Dev 9: 422-426. 


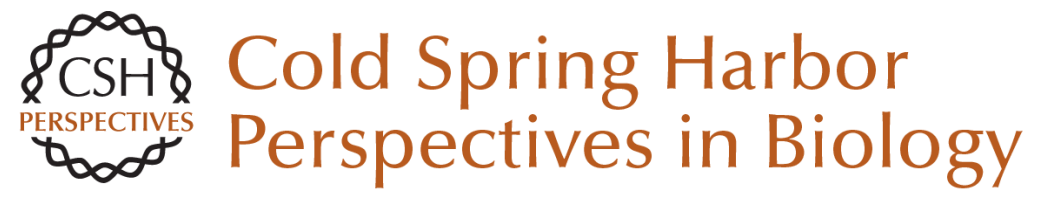

\section{Left-Right Determination: Involvement of Molecular Motor KIF3, Cilia, and Nodal Flow}

Nobutaka Hirokawa, Yosuke Tanaka and Yasushi Okada

Cold Spring Harb Perspect Biol 2009; doi: 10.1101/cshperspect.a000802

Subject Collection Symmetry Breaking in Biology

Cytoskeletal Mechanisms for Breaking Cellular Symmetry

R. Dyche Mullins

Symmetry Breaking in Biology

Rong Li and Bruce Bowerman

Planar Cell Polarity Signaling: The Developing Cell's Compass

Eszter K. Vladar, Dragana Antic and Jeffrey D. Axelrod

Cellular Polarity in Prokaryotic Organisms Jonathan Dworkin

Symmetry Breaking in Plants: Molecular Mechanisms Regulating Asymmetric Cell Divisions in Arabidopsis

Jalean J. Petricka, Jaimie M. Van Norman and Philip N. Benfey

The Signaling Mechanisms Underlying Cell

Polarity and Chemotaxis

Fei Wang

Polarization of Drosophila Neuroblasts During

Asymmetric Division Kenneth E. Prehoda

Physical Model of Cellular Symmetry Breaking Jasper van der Gucht and Cécile Sykes
Polarity in Stem Cell Division: Asymmetric Stem

Cell Division in Tissue Homeostasis

Yukiko M. Yamashita, Hebao Yuan, Jun Cheng, et al.

Symmetry Breaking in the Life Cycle of the

Budding Yeast

Brian D. Slaughter, Sarah E. Smith and Rong Li

Neuronal Polarity

Sabina Tahirovic and Frank Bradke

Membrane Organization and Dynamics in Cell Polarity

Kelly Orlando and Wei Guo

Cellular Symmetry Breaking during

Caenorhabditis elegans Development

Edwin Munro and Bruce Bowerman

Symmetry Breaking During Drosophila Oogenesis Siegfried Roth and Jeremy A. Lynch

Widely Conserved Signaling Pathways in the Establishment of Cell Polarity

Luke Martin McCaffrey and lan G. Macara

Shaping Fission Yeast with Microtubules Fred Chang and Sophie G. Martin

For additional articles in this collection, see http://cshperspectives.cshlp.org/cgi/collection/

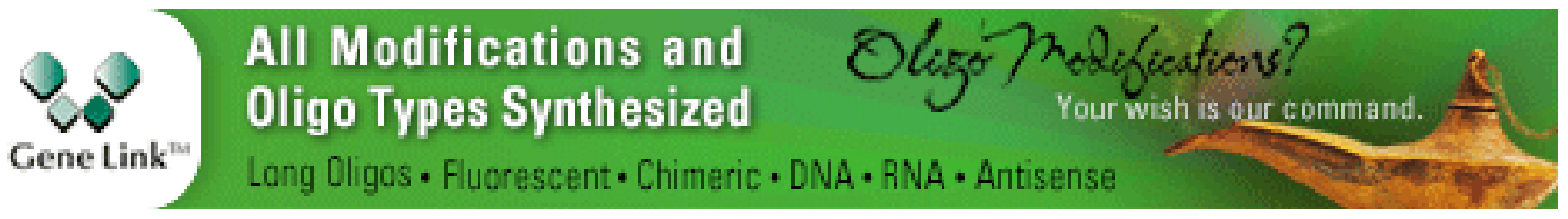

